\title{
Model of Intraperitoneal Targeted $\alpha$-Particle Therapy Shows That Posttherapy Cold-Antibody Boost Enhances Microtumor Radiation Dose and Treatable Tumor Sizes
}

\author{
Stig Palm ${ }^{1}$, Tom Bäck ${ }^{1}$, Sture Lindegren ${ }^{1}$, Ragnar Hultborn ${ }^{2}$, Lars Jacobsson ${ }^{1}$, and Per Albertsson ${ }^{2}$ \\ ${ }^{1}$ Department of Radiation Physics, Institute for Clinical Sciences, Sahlgrenska Academy at the University of Gothenburg, \\ Gothenburg, Sweden; and ${ }^{2}$ Department of Oncology, Institute for Clinical Sciences, Sahlgrenska Academy at the University of \\ Gothenburg, Gothenburg, Sweden
}

\begin{abstract}
Intraperitoneally administered radiolabeled monoclonal antibodies (mAbs) have been tested in several clinical trials, often with promising results, but have never proven curative. Methods: We have previously presented simulations of clinically relevant amounts of intraperitoneal ${ }^{90} \mathrm{Y}-\mathrm{mAbs}$ for treatment of minimal disease and shown that such treatments are unlikely to eradicate microtumors. Our previous model simulated the kinetics of intraperitoneally infused radiolabeled mAbs in humans and showed the benefit of instead using a-emitters such as ${ }^{211} \mathrm{At}$. In the current work, we introduce penetration of mAbs into microtumors with radii of up to $400 \mu \mathrm{m}$. Calculations were performed using dynamic simulation software. To determine the radiation dose distribution in nonvascularized microtumors of various sizes after intraperitoneal ${ }^{211} \mathrm{At}-$ radioimmunotherapy, we used an in-house-developed Monte Carlo program for microdosimetry. Our aim was to find methods that optimize the therapy for as wide a tumor size range as possible. Results: Our results show that high-specific-activity radiolabeled $\mathrm{mAbs}$ that are bound to a tumor surface will penetrate slowly compared with the half-lives of ${ }^{211} \mathrm{At}$ and shorter-lived radionuclides. The inner-core cells of tumors with radii exceeding $100 \mu \mathrm{m}$ may therefore not be sufficiently irradiated. For lower specific activities, the penetration rate and dose distribution will be more favorable for such tumors, but the dose to smaller microtumors and single cells will be low. Conclusion: Our calculations show that the addition of a boost with unlabeled $\mathrm{mAb} 1-5 \mathrm{~h}$ after therapy results in sufficient absorbed doses both to single cells and throughout microtumors up to approximately $300 \mu \mathrm{m}$ in radius. This finding should also hold for other high-affinity mAbs and short-lived a-emitters.
\end{abstract}

Key Words: alpha-particle therapy; biokinetic modeling; ${ }^{211} \mathrm{At}$; radioimmunotherapy; ovarian cancer

J Nucl Med 2018; 59:646-651

DOI: 10.2967/jnumed.117.201285

I ntraperitoneally administered radiolabeled monoclonal antibodies (mAbs) to treat epithelial ovarian cancer have been tested in several clinical trials, initially with promising results, but in

Received Aug. 25, 2017; revision accepted Nov. 6, 2017.

For correspondence or reprints contact: Stig Palm, Department of Radiation Physics, University of Gothenburg, Gula Stråket 2b, SE-413 45 Gothenburg, Sweden.

E-mail: stig.palm@gu.se

Published online Nov. 24, 2017.

COPYRIGHT (C 2018 by the Society of Nuclear Medicine and Molecular Imaging. phase III randomized trials have never proven curative $(1,2)$. Most studies have involved $\beta$-emitters with relatively long half-lives (on the order of days) and ranges (on the order of millimeters in tissue). Although these characteristics might provide relatively high absorbed doses to larger tumors, they also result in irradiation of normal tissue, leading to toxicity (3). This limits the amount that can be administered, and curative absorbed doses to microtumors have not been achieved.

Previously, we presented simulations of clinically relevant amounts of intraperitoneal ${ }^{90} \mathrm{Y}$-mAbs for treatment of minimal disease and showed that such treatments are unlikely to eradicate microtumors with diameters of less than approximately $100 \mu \mathrm{m}$ (4). That same model showed the benefit of instead using $\alpha$-emitters such as ${ }^{211} \mathrm{At}$, which we applied in early clinical trials (5), or ${ }^{212} \mathrm{~Pb}$, as reported by Meredith et al. (6). We have also demonstrated the therapeutic effect in several studies involving a mouse model with disseminated microscopic tumors $(7,8)$.

However, we have also observed in experimental models that tumors estimated to have radii larger than about $100 \mu \mathrm{m}$ can resist $\alpha$-emitter-based treatments (8). The difficulty of eradicating larger tumors stems from the short half-life of ${ }^{211}$ At in comparison with the penetration rate of mAbs into tumor tissue. Most ${ }^{211}$ At decays will therefore occur on the surface of the microtumors, and the approximately $70-\mu \mathrm{m}$ range of the emitted $\alpha$-particles will limit the irradiation depth. ${ }^{211} \mathrm{At}-\mathrm{mAb}$ concentrations that are high in the outer shell but low in the center of larger microtumors have also been experimentally observed (9).

Penetration of antibodies into tumor tissue has previously been described, modeled, and experimentally studied using spheroids (10-13). According to these models, diffusion is hindered by the so-called binding barrier. The penetration rate is derived from the molecule's diffusion coefficient but also depends on antigen density and the antibody's binding characteristics and concentration over time. Using these models, it was recently shown (14) that intraperitoneal tumors are better accessed by intraperitoneal administration than by intravenous administration. This was found also for vascularized tumors and for administration of relatively large molecules. These results justify further investigation of possible improvements in intraperitoneal radioimmunotherapy.

In the current work, we have used model calculations, based on human data, to determine the radiation dose distribution in nonvascularized microtumors of various sizes after intraperitoneal ${ }^{211}$ At radioimmunotherapy. Our aim was to find methods that optimize the therapy for as wide a tumor size range as possible. 


\section{MATERIALS AND METHODS}

The current calculation model is a development of our previously presented model for the kinetics of intraperitoneally infused ${ }^{211} \mathrm{At}-$ labeled $\mathrm{mAb}$ in humans (4). That model included transport of mAbs from the peritoneal cavity and binding of mAbs to the surface of spheric microscopic tumors. In the current work, we introduce penetration of mAbs into microtumors with radii of up to $400 \mu \mathrm{m}$. The penetration calculations were based on the binding barrier theory presented by Fujimori et al. (10) and applied for spheric microtumors (12).

All results are based on a clinical situation in which therapy is administered intraperitoneally with $300 \mathrm{MBq}$ of ${ }^{211}$ At-labeled mAb in $1.7 \mathrm{~L}$ of $7.5 \%$ icodextrin (5). The optimizations are based on the binding kinetics of mAbs to easily accessible antigens. This binding condition is found in vivo for single cells and for the outer layers of small tumors, such as microtumors. Unlabeled and radiolabeled mAbs are assumed to exhibit identical kinetics. The easily accessible antigens are modeled as directly exposed to the intraperitoneal fluid. The binding to these antigens depends on the affinity constant (on-rate, or $k_{o n}$ ) for the antibodies and the time-dependent concentration of mAbs in the intraperitoneal fluid.

Calculations were performed by dynamic simulation using the software Stella (ISEE Systems, Inc.). The radial net transport of mAbs was assumed to follow Fick's law. During the time interval $\Delta t$, the number of mAbs transferred from shell $i$ to $(i+1)$ is given by...

$$
\Delta n_{i}(t)=\frac{\Delta t \cdot D \cdot A_{i} \cdot\left\{C_{i}(t)-C_{i+1}(t)\right\}}{\Delta x} .
$$

Antibody binding to antigen in shell $i$ is described by...

$$
\Delta n_{b i}=\Delta t \cdot\left(n_{i}(t) \cdot k_{o n} \cdot \frac{n_{a g, i}(t)}{V_{i} \cdot f}-n_{b i}(t) \cdot k_{o f f}\right) .
$$

The parameters are described in Table 1, and the values used in our simulations are found in Table 2. The values for $k_{\text {on }}$ and for the offrate, $k_{\text {off }}$, were set for the MX35 mAb (4). $k_{o f f}$, at less than $3 \% \mathrm{~h}^{-1}$, was approximated to be zero.

Binding to antigens on single-cell and microtumor surfaces was assumed to occur without any hindrance because of the direct exposure to the intraperitoneal-fluid antibody concentration. These antigens are referred to as "easily accessible" throughout this work. The antigen density, that is, the number of antigens per surface area, for various sizes of microtumor was set to be the same as that found for a single cell. Our default setting was 700,000 antigens on a 7- $\mu \mathrm{m}-$ radius cell, resulting in an antigen density of $1,137 \mu \mathrm{m}^{-2}$. When modeling microtumors, the volumes of the tumor cells remained

\section{TABLE 1}

Description of Parameters used in Equations 1 and 2

\begin{tabular}{ll}
\hline Parameter & \multicolumn{1}{c}{ Description } \\
\hline$V_{i}$ & Shell volume \\
$A_{i}$ & Intersection area between shells \\
$n_{i}(t)$ & Number of free mAbs in shell $i$ \\
$C_{i}(t)$ & Antibody concentration in shell $i$ \\
$n_{\text {ag, } i(t)}(t)$ & Number of free antigens in shell $i$ \\
$\Delta n_{i}(t)$ & Number of transferred mAbs \\
$n_{b i}(t)$ & Number of bound mAbs in shell $i$ \\
\hline
\end{tabular}

TABLE 2

Parameter Values Used for the Presented Simulations

\begin{tabular}{ll}
\hline \multicolumn{1}{c}{ Parameter } & \multicolumn{1}{c}{ Value } \\
\hline Administered activity & $300 \mathrm{MBq}$ of ${ }^{211} \mathrm{At}$ \\
\hline Administered fluid & $1.7 \mathrm{~L}$ of icodextrin \\
Fraction of labeled mAbs & 1 in $200 \mathrm{mAbs}$ radiolabeled \\
$D$ & $14 \mu^{2} \mathrm{~s}^{-1}(21)$ \\
$f$ (fraction fluid in tumor) & $0.1(11)($ cell-packing ratio, $1-f)$ \\
$\Delta t$ & $0.5 \mathrm{~s}$ \\
$\Delta x$ & 5 or $10 \mu \mathrm{m}$ \\
$k_{\text {on }}$ & $44,000 \mathrm{M}^{-1} \mathrm{~s}^{-1}(4)$ \\
$k_{\text {off }}$ & 0 \\
Antigens per cell & $700,000(4)$ \\
\hline
\end{tabular}

constant, but the shapes of individual cells were allowed to change so that a packing ratio higher than that found for packed spheres was achieved. The antigen concentration, that is, the number of antigens per volume, of the tumor cells was $487 \mu \mathrm{m}^{-3}$. This number was then reduced with the packing ratio, reflecting the microtumor tissue that is not tumor cells. For a fraction of fluid in the tumor volume of $f=0.1$, all studied microtumor sizes were given an antigen concentration of $438 \mu \mathrm{m}^{-3}$, homogeneously distributed throughout the tumor volume.

The radiolabeled compound is described by the specific activity. This is often expressed as the radioactivity $(\mathrm{Bq})$ of the radionuclide per amount $(\mathrm{mg})$ of $\mathrm{mAb}$. To better illustrate the significance of specific activity, we instead chose to express it in terms of the fraction of radiolabeled $\mathrm{mAb}$ (i.e., mAbs with a bound radioactive atom) to unlabeled $\mathrm{mAb}$. Throughout this work, we therefore express specific activity as, for example, 1 in $200 \mathrm{mAbs}$ being radiolabeled, 1 in 1,000 $\mathrm{mAbs}$ radiolabeled, and so on. The number of mAbs and the specific activity then allow calculation of cumulated activity, that is, the total number of radionuclide decays. This was performed in each $5-\mu \mathrm{m}$ tumor shell (sphere radius, $\leq 200 \mu \mathrm{m}$ ) or $10-\mu \mathrm{m}$ tumor shell (sphere radius, $>200 \mu \mathrm{m}$ ) and was done for $50 \mathrm{~h}$, when more than $99 \%$ of all ${ }^{211}$ At atoms have decayed. A flow chart is presented as Supplemental Figure 1 (supplemental materials are available at http://jnm. snmjournals.org).

For microdosimetry, an in-house-developed Monte Carlo program (15) was used. The decays were positioned homogeneously within a particular tumor shell, and the resulting specific energy to each tumor shell was recorded. This procedure was repeated for decays occurring in all tumor shells. The weighted results provided the absorbed dose to each shell of microtumors with radii of 100, 200, 300, and $400 \mu \mathrm{m}$. For the single cell (radius, $7.0 \mu \mathrm{m}$ ), the energy imparted to the cell nucleus (radius, $5.6 \mu \mathrm{m}$ ) was calculated.

\section{RESULTS}

The kinetics of mAb binding to the easily accessible antigens is illustrated in Supplemental Figure 2 for 2 values of $k_{\text {on }}, 100,000$ and the $44,000 \mathrm{M}^{-1} \mathrm{~s}^{-1}$ valid for our clinically studied $\mathrm{mAb}$. Ninety percent of maximum binding, a percentage at which all easily accessible antigens are occupied by mAbs, is reached after 2.5 and $7.5 \mathrm{~h}$ for these $k_{\text {on }}$ values of 100,000 and the $44,000 \mathrm{M}^{-1} \mathrm{~s}^{-1}$, respectively. The results were derived for 300 $\mathrm{MBq}$ of ${ }^{211} \mathrm{At}-\mathrm{mAb}$ with a specific activity of 1 in $200 \mathrm{mAbs}$ radiolabeled. Despite the relatively low intraperitoneal-fluid antibody concentration, almost all easily accessible antigens will be 


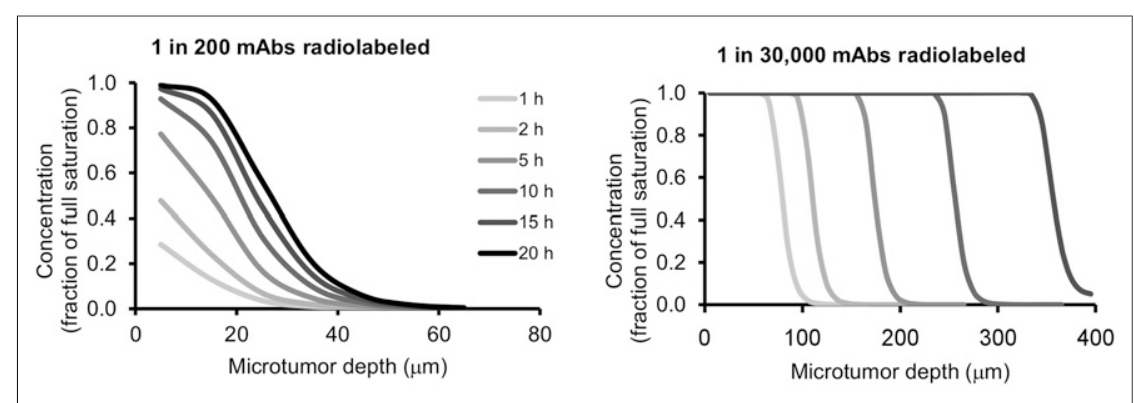

FIGURE 1. Simulated penetration of mAbs in microtumor after intraperitoneal infusion of radiolabeled $\mathrm{mAb}$. Concentration of $\mathrm{mAb}$ is shown at different depths in microtumors at various times after intraperitoneal infusion of $300 \mathrm{MBq}$ of ${ }^{211} \mathrm{At}-\mathrm{mAb}$ of relatively high specific activity (1 in $200 \mathrm{mAbs}$ radiolabeled) and low specific activity ( 1 of $30,000 \mathrm{mAbs}$ radiolabeled). Concentration is normalized to that of complete saturation-that is, when all antigens in that shell are occupied by a mAb.

bound within a few hours, provided that $k_{\text {off }}$ is negligible. This is also true for our studied MX35 mAb (16), despite its lower $k_{\text {on }}$. The general results are valid for any amount of easily accessible antigens-that is, on cells with varying antigen expression.

Since the range of emitted $\alpha$-particles is approximately $70 \mu \mathrm{m}$ in tissue, and since the easily accessible antigens are on the surface of microtumors, successful $\alpha$-particle irradiation of tumors with radii larger than approximately $50 \mu \mathrm{m}$ relies on $\mathrm{mAb}$ diffusion to the subsurface layers of such tumors.

It is often the amount of administered radioactivity that sets the limit for targeted radiotherapies, because of the risk of healthyorgan radiotoxicity. Diffusion of mAbs in microtumors, however, depends only on the intraperitoneal-fluid mAb concentration. This concentration will differ for the same amount of radioactivity if the specific activity of the conjugate is varied.

Figure 1 shows the expected time-dependent $\mathrm{mAb}$ tissue concentration at various microtumor depths for 2 different specific activities of $300-\mathrm{MBq}^{211}$ At-labeled mAb: a high specific activity corresponding to 1 in $200 \mathrm{mAbs}$ radiolabeled, and a low specific activity corresponding to 1 in $30,000 \mathrm{mAbs}$ radiolabeled. For this fixed amount of radioactivity, the low-specific-activity example thus contains 150 times more mAb. Such a high concentration results in rapid binding of the easily accessible antigens on the microtumor surface, and virtually all such antigens are occupied by a mAb in less than 5 min after intraperitoneal infusion. Such saturation effectively makes antigens in deeper layers of microtumors accessible for binding, and the binding barrier shifts inward when there is an excess of mAbs in
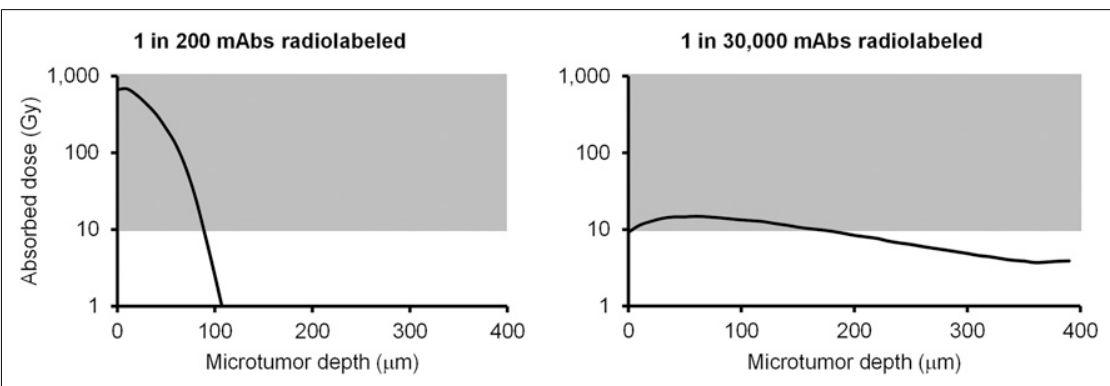

FIGURE 2. Absorbed doses at various depths of 400- $\mu \mathrm{m}$-radius microtumor after intraperitoneal infusion of $300 \mathrm{MBq}$ of ${ }^{211} \mathrm{At}-\mathrm{mAb}$ of relatively high specific activity (1 of $200 \mathrm{mAbs}$ radiolabeled) and low specific activity (1 of 30,000 mAbs radiolabeled). the surrounding fluid. The high-specificactivity example illustrates how (the relatively few) mAbs bind almost exclusively to the easily available antigens on the microtumor surface.

For a single cell, irradiation with a high specific activity ( 1 in 200 mAbs radiolabeled) leads to an absorbed dose to its nucleus of $60 \mathrm{~Gy}$. For a specific activity corresponding to 1 in $1,000 \mathrm{mAbs}$ radiolabeled, the nucleus receives 15 Gy. For a low specific activity ( 1 in $30,000 \mathrm{mAbs}$ radiolabeled), the nucleus receives only $0.53 \mathrm{~Gy}$. These results show that a relatively high specific activity is necessary to eradicate single cells and the smallest microtumors. If larger microtumors are present, sufficient irradiation of their core can be achieved only if the administered $\mathrm{mAb}$ amount is drastically increased. Such intentional lowering of the specific activity will, however, result in an absorbed dose insufficient to eradicate the single cells. Figure 2 shows the absorbed dose to various layers of a $400-\mu \mathrm{m}$-radius microtumor exposed to intraperitoneally infused $300-\mathrm{MBq}$ high- and low-specific-activity ${ }^{211}$ At-mAbs without a subsequent boost. The different mAb concentrations result in both different amounts of radioactivity in the tumors and different dose distributions. Figure 3 shows the absorbed dose distribution for various microtumor sizes.

To circumvent the apparent limitations of infusing high or low specific activities, we propose using a 2-step procedure. If a highspecific-activity ${ }^{211} \mathrm{At}-\mathrm{mAb}$ intraperitoneal infusion is followed, at some later time, by a much higher amount of unlabeled $\mathrm{mAb}$, then sufficient absorbed doses to a range of microtumor sizes can be achieved.

The basis for optimizing a 2-step procedure is the mAb-binding kinetics onto easily accessible antigens (Supplemental Fig. 2). The shorter the time from first infusion to delivery of boost, the more ${ }^{211} \mathrm{At}-\mathrm{mAb}$ in the intraperitoneal fluid will be available for binding to antigens in the inner shells of microtumors. Figure 4 shows the resulting absorbed dose distribution in microtumors when a boost with 150 times the $\mathrm{mAb}$ amount of the first infusion is delivered 1 or $5 \mathrm{~h}$ after the first infusion. After this posttherapy coldantibody boost, a much smaller mAb fraction will carry an astatine atom, and the number of ${ }^{211} \mathrm{At}-\mathrm{mAbs}$ bound to the easily accessible antigens will be reduced.

For the conditions used in Figure 4, a single cell will receive 22 or 52 Gy following boost 1 or $5 \mathrm{~h}$ after therapy, respectively. A longer delay before delivery of the boost will thus result in a higher dose to the smallest tumors but, because of radionuclide decay, a somewhat lower dose to the core of larger microtumors. Further optimization of the delay before the boost is therefore not crucial because similar results can be expected for boosts delivered between 1 and $5 \mathrm{~h}$ after therapy.

\section{DISCUSSION}

For intraperitoneal radioimmunotherapy, we have previously shown that to achieve eradicative radiation doses to microtumors 


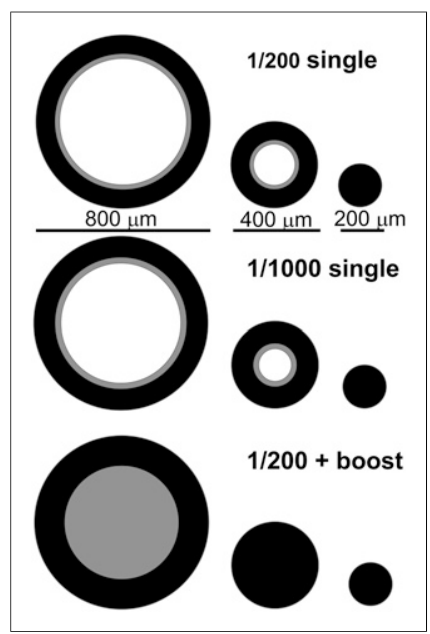

FIGURE 3. Absorbed dose distribution for microtumors with radii of 400, 200, and $100 \mu \mathrm{m}$. Each graph is illustrated by mid-sphere section, with gray-scale-coded shells representing likely eradicative absorbed doses (>10 Gy, black), likely insufficient absorbed doses ( $<1$ Gy, white), and absorbed doses for which biologic outcome is less certain (gray).

The model we applied for antibody penetration has been used to mathematically describe the so-called binding barrier. This phenomenon occurs because mAb binds to easily accessible antigen in the outer shells and is thereby hindered from diffusing freely. It is only when these antigens begin to be occupied by mAbs that excess $\mathrm{mAb}$ can diffuse past these layers. The transfer of a mAb in the tumor intercellular space will thus be determined by both diffusion and its probability of binding to an available antigen. Others have used numeric solutions of coupled differential equations to describe this transfer $(10,11,14)$. In the current work, we instead used dynamic simulation software, Stella, for our model calculations. The program simulates the diffusion and binding of mAbs for a short interval $(0.5 \mathrm{~s})$, repeated with a stepwise movement of time for up to $50 \mathrm{~h}$ after administration. After that time, less than $1 \%$ of the radioactivity remains.

Because the interval was set to be short, the mAb concentration in each tumor shell did not significantly change and could be set constant for the duration of each interval. Predicting mAb transfer within each interval was then a matter of solving equations based on Fick's law and the basic antibody-antigen binding process. The mAb concentration of the intraperitoneal fluid outside the tumors for each interval was calculated using our previously presented program that describes the time-dependent intraperitoneal concentration of $\mathrm{mAb}(4)$. Combining the change in intraperitoneal-fluid $\mathrm{mAb}$ concentration with the expected transport within tumors allowed us to simulate the effect of multiple administrations, which, in the current work, was shown to be promising.

The results were generated for an antibody that we used in a clinical trial (5) and for which we have extensive preclinical data. The aim was to optimize the intraperitoneal ${ }^{211}$ At radioimmunotherapy so that it could be expected to eradicate a broad range of microtumor sizes while keeping the eventual systemic irradiation as low as possible. The calculations were therefore based on an administered activity concentration of about $200 \mathrm{MBq} / \mathrm{L}$. This concentration was estimated to provide an acceptably low total-body irradiation (17). Long-term detrimental effects are of particular concern because our treatment is aimed at an adjuvant setting in which about $30 \%$ of the patients are expected to be tumor-free after standard care.

Some biologic variables that might alter the results were not included in the model. These include heterogeneity of receptor expression within the microtumor, rate of antibody deastatination in vivo, and possible receptor-antibody internalization kinetics and subsequent subcellular localization. It was also beyond the scope of this current work to detail the expected radiosensitivity, dose microdistribution, and possible bystander effects from $\alpha$-particle irradiation. A tumor control probability, TCP, analysis of cells in each tumor shell could possibly better predict the fate, but the uncertainties of the results would be significant. Instead we have, throughout this current work, used
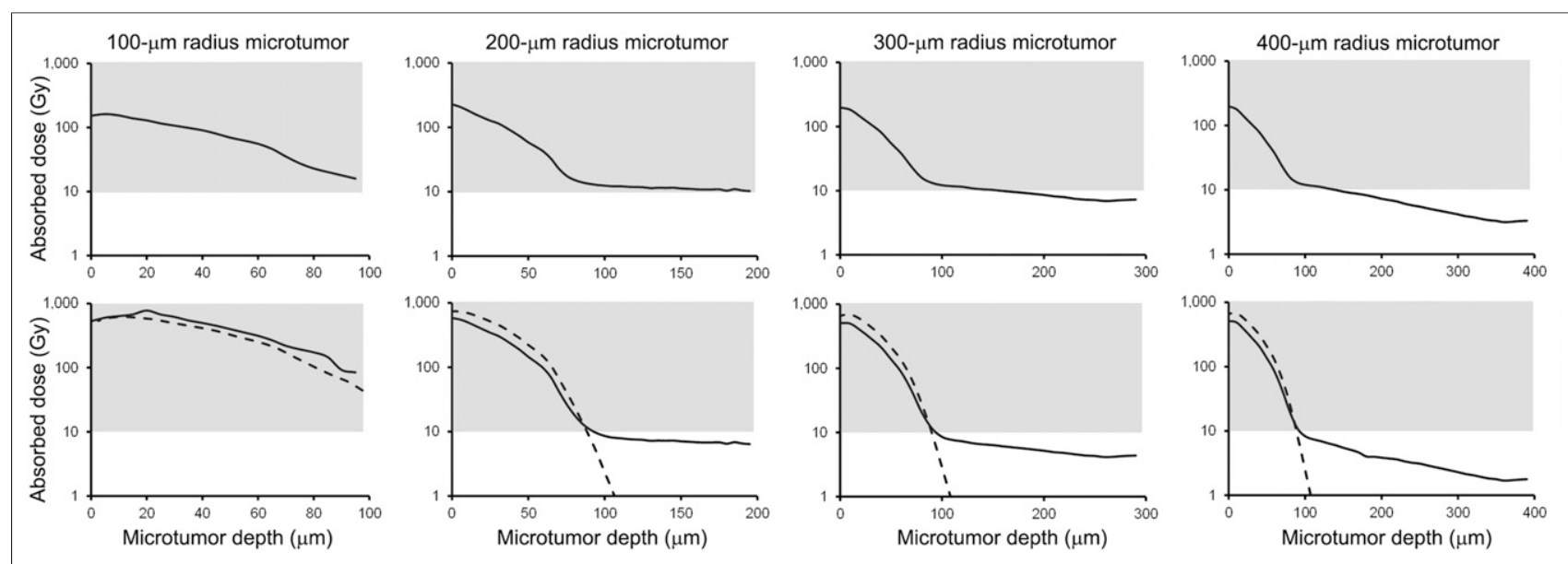

FIGURE 4. Absorbed dose distribution to spheric tumors with radii of $100,200,300$, and $400 \mu \mathrm{m}$ after intraperitoneal infusion of high-specificactivity ${ }^{211} \mathrm{At}-\mathrm{mAb}$ ( 1 in $200 \mathrm{mAbs}$ radiolabeled) followed by boost with 150 times mAb amount of first infusion. Shown are results when posttherapy cold-antibody boost is delivered $1 \mathrm{~h}$ after therapy (top) and $5 \mathrm{~h}$ after therapy (bottom). Dashed line shows results without posttherapy cold-antibody boost. 
a threshold of 10 Gy to describe an eradicative dose. This choice is partly based on our previous work, which showed that cure of subcutaneous tumors from ${ }^{211}$ At-MX35 irradiation was achieved at $10 \mathrm{~Gy}$ (18). Although this dose carries large uncertainties when used for microtumors and single cells, we have chosen to use it to discuss how intraperitoneal ${ }^{211}$ At radioimmunotherapy can be optimized.

Improvements in radiolabeling techniques make it possible to label mAbs with ${ }^{211}$ At to a high specific activity (19). In our clinical trial (17), up to 1 in 200 mAbs were radiolabeled. This ratio was also used for the simulations presented in the current work. High specific activity is required to achieve high absorbed doses to isolated single cells and very small cell aggregates (4). However, because the mAb concentration is then low, the time for $\mathrm{mAb}$ transport to deeper, embedded, layers of antigens in larger tumors will be long. Hence, a lower specific activity could result in a better, that is, shallower, dose profile. The 10-Gy front would reach deeper and the surface dose would be slightly lower (but still higher than $10 \mathrm{~Gy}$ ) for a lower specific activity than for a higher specific activity. For our default assumptions, a specific activity higher than 1 in 1,000 mAbs radiolabeled is needed for eradication of isolated single cells. If the specific activity were intentionally lowered from 1 in 200 to 1 in 1,000 mAbs radiolabeled, however, the 10-Gy front would move only a little, from 89 to $102 \mu \mathrm{m}$ in depth, in 200- $\mu$ m-radius spheric microtumors. Such microtumors will thus not be sufficiently irradiated. Instead, a seminal improvement in irradiating such microtumors is made by adding a boost infusion of unlabeled mAb. Such a postherapy cold-antibody boost will cause all tumor cells to receive an absorbed dose of more than $10 \mathrm{~Gy}$.

The results are presented for different sizes of microtumor and a boost using a 150-times-higher unlabeled-mAb amount. Increasing the boost amount generally helps the irradiation also reach the core of larger microtumors. The optimal amount to reach the 10-Gy level for the studied microtumor sizes is 150 times. In a clinical setting, however, the optimal boost amount is difficult to establish, partly because the distribution of microtumor sizes in the individual patient is unknown.

Binding to easily accessible antigen is relatively rapid. In our default setting, around $80 \%$ of such antigens will be occupied within $5 \mathrm{~h}$. However, unlabeled mAbs delivered in a boost will compete for the same antigens. Therefore, if the boost is delivered too early, the smallest tumors or single cells might not have bound enough ${ }^{211}$ At-labeled mAb. A delay of a few hours will reduce this effect, but at a cost of reducing the depth-dose by about $9 \%$ per hour, because of the physical half-life of ${ }^{211}$ At. A boost delivered within the proper time should, however, not significantly decrease the ability to eliminate the smallest tumors and single cells. Hence, there is no obvious disadvantage to incorporating a posttherapy cold-antibody boost. Adding a boost is also relatively straightforward and should not have a significant impact on patient comfort or staff logistics.

Our default setting uses an antigen expression of 700,000 per cell. A lower antigen expression will typically result in lower absorbed doses. Penetration is, however, more rapid because fewer sites need to be filled before saturation occurs and antigens become accessible to deeper microtumor layers. This effect mediates the effect of the lower absorbed dose in that a somewhat better dose profile is achieved. In general, our calculations show that variations of up to $50 \%$ in antigen expression do not alter our conclusions on optimal treatment methods.
In addition to antigen binding, the diffusion coefficient determines the penetration. This coefficient is uncertain, with reported values of around $10 \mu \mathrm{m}^{2} / \mathrm{s}$ for $\mathrm{IgG}$ in tumor tissue (20). We use a coefficient of $14 \mu \mathrm{m}^{2} / \mathrm{s}(21)$. For $\mathrm{F}\left(\mathrm{ab}^{\prime}\right)_{2}$, used in our clinical study with MX35, theoretically the coefficient is around $15 \%$ greater than for $\operatorname{IgG}(22)$. However, this uncertainty in the diffusion coefficient does not affect the major findings of this current work. A $\pm 50 \%$ change in the used coefficient results in moving of the 10 -Gy front by $\pm 10 \mu \mathrm{m}$ in $200-\mu \mathrm{m}$-radius microtumors. For molecules much smaller than $\mathrm{F}\left(\mathrm{ab}^{\prime}\right)_{2}$, such as singlechain antibody fragments, the diffusion coefficient is significantly higher, at around $80 \mathrm{\mu m}^{2} / \mathrm{s}$ (11), and eradicative doses can be achieved throughout spheric microtumors with radii of up to $200 \mu \mathrm{m}$ without a boost, as long as the binding characteristics are intact. For larger tumors, a boost will be required for such small molecules.

To further optimize intraperitoneal radionuclide therapy, additional radionuclides might also be considered. We have previously addressed the difficulty of eradicating microtumors within tolerable toxicity for various $\beta$-emitting radionuclides (4). $\alpha$-emitters, therefore, remain our main candidates. Those with longer halflives than ${ }^{211}$ At would, in some situations, result in a higher probability of cure. Examples such as ${ }^{212} \mathrm{~Pb}$ and ${ }^{225} \mathrm{Ac}$ are, however, often considered unsuitable because their complex decay chain includes $\alpha$-emitting daughter nuclides that likely increase normalorgan toxicity. A boost might, however, cause the parent decay to occur at some depth within the tumor, adding to the likelihood that the daughter nuclides also will decay within the tumor. This possibility should be further investigated.

\section{CONCLUSION}

The modeling shows that 1 -step intraperitoneal $\alpha$-particle radioimmunotherapy is unlikely to provide sufficient irradiation of all the sizes of microtumor expected in a minimal disease setting. High-specific-activity radiolabeled high-affinity mAbs that are bound to a tumor surface will penetrate more slowly than the half-lives of ${ }^{211}$ At and shorter-lived radionuclides. The inner-core cells of tumors with radii exceeding $100 \mu \mathrm{m}$ may therefore not be sufficiently irradiated. For lower specific activities, the penetration rate and dose distribution will be more favorable for such tumors, but the dose to smaller microtumors and single cells will be low.

Our calculations show that a boost with unlabeled mAb 1-5 h after therapy results in eradicative doses both to single cells and throughout microtumors of up to about $300 \mu \mathrm{m}$ in radius. On the basis of our modeling, we therefore suggest the use of a posttherapy cold-antibody boost at 1-5 h after high-specific-activity therapy. This suggestion should also be valid for other highaffinity mAbs and short-lived $\alpha$-emitters. The addition of a posttherapy cold-antibody boost carries minimal risk, is clinically feasible, and facilitates sufficient irradiation of all relevant sizes of microtumor.

\section{DISCLOSURE}

Financial support was provided by the Swedish Research Council, the Swedish Cancer Society, and the King Gustav V Jubilee Clinic Research Foundation and through the regional agreement on medical training and clinical research (ALF). No other potential conflict of interest relevant to this article was reported. 


\section{REFERENCES}

1. Nicholson S, Bell SJ, McCormack M, et al. A randomized phase III trial of adjuvant intraperitoneal radioimmunotherapy in ovarian cancer [abstract]. Proc Am Soc Clin Oncol. 2000;19:383.

2. Verheijen RH, Massuger LF, Benigno BB, et al. Phase III trial of intraperitoneal therapy with yttrium-90-labeled HMFG1 murine monoclonal antibody in patients with epithelial ovarian cancer after a surgically defined complete remission. J Clin Oncol. 2006;24:571-578.

3. Elgqvist J, Frost S, Pouget JP, Albertsson P. The potential and hurdles of targeted alpha therapy: clinical trials and beyond. Front Oncol. 2014;3:324.

4. Palm S, Back T, Haraldsson B, Jacobsson L, Lindegren S, Albertsson P. Biokinetic modeling and dosimetry for optimizing intraperitoneal radioimmunotherapy of ovarian cancer microtumors. J Nucl Med. 2016;57:594-600.

5. Andersson H, Cederkrantz E, Back T, et al. Intraperitoneal alpha-particle radioimmunotherapy of ovarian cancer patients: pharmacokinetics and dosimetry of ${ }^{211}$ At-MX35 F(ab')2—a phase I study. J Nucl Med. 2009;50:1153-1160.

6. Meredith R, Torgue J, Shen S, et al. Dose escalation and dosimetry of first-inhuman alpha radioimmunotherapy with ${ }^{212} \mathrm{~Pb}$-TCMC-trastuzumab. J Nucl Med. 2014;55:1636-1642.

7. Andersson H, Elgqvist J, Horvath G, et al. Astatine-211-labeled antibodies for treatment of disseminated ovarian cancer: an overview of results in an ovarian tumor model. Clin Cancer Res. 2003;9(suppl):3914S-3921S.

8. Elgqvist J, Andersson H, Back T, et al. Alpha-radioimmunotherapy of intraperitoneally growing OVCAR-3 tumors of variable dimensions: outcome related to measured tumor size and mean absorbed dose. J Nucl Med. 2006;47:1342-1350.

9. Chouin N, Lindegren S, Jensen H, Albertsson P, Back T. Quantification of activity by alpha-camera imaging and small-scale dosimetry within ovarian carcinoma micrometastases treated with targeted alpha therapy. Q J Nucl Med Mol Imaging. 2012;56:487-495.

10. Fujimori K, Covell DG, Fletcher JE, Weinstein JN. Modeling analysis of the global and microscopic distribution of immunoglobulin $\mathrm{G}, \mathrm{F}\left(\mathrm{ab}^{\prime}\right) 2$, and Fab in tumors. Cancer Res. 1989;49:5656-5663.

11. Graff CP, Wittrup KD. Theoretical analysis of antibody targeting of tumor spheroids: importance of dosage for penetration, and affinity for retention. Cancer Res. 2003;63:1288-1296.
12. van Osdol W, Fujimori K, Weinstein JN. An analysis of monoclonal antibody distribution in microscopic tumor nodules: consequences of a "binding site barrier." Cancer Res. 1991;51:4776-4784.

13. Weinstein JN, van Osdol W. Early intervention in cancer using monoclonal antibodies and other biological ligands: micropharmacology and the "binding site barrier." Cancer Res. 1992;52(suppl):2747s-2751s.

14. Winner KK, Steinkamp MP, Lee RJ, et al. Spatial modeling of drug delivery routes for treatment of disseminated ovarian cancer. Cancer Res. 2016;76:13201334.

15. Palm S, Humm JL, Rundqvist R, Jacobsson L. Microdosimetry of astatine-211 single-cell irradiation: role of daughter polonium-211 diffusion. Med Phys. 2004; 31:218-225.

16. Finstad CL, Lloyd KO, Federici MG, et al. Distribution of radiolabeled monoclonal antibody MX35 $\mathrm{F}\left(\mathrm{ab}^{\prime}\right) 2$ in tissue samples by storage phosphor screen image analysis: evaluation of antibody localization to micrometastatic disease in epithelial ovarian cancer. Clin Cancer Res. 1997;3:14331442 .

17. Cederkrantz E, Andersson H, Bernhardt P, et al. Absorbed doses and risk estimates of ${ }^{211}$ At-MX35 $\mathrm{F}\left(\mathrm{ab}^{\prime}\right) 2$ in intraperitoneal therapy of ovarian cancer patients. Int J Radiat Oncol Biol Phys. 2015;93:569-576.

18. Bäck T, Chouin N, Lindegren S, et al. Cure of human ovarian carcinoma solid xenografts by fractionated alpha-radioimmunotherapy with ${ }^{211}$ At-MX35-F(ab') 2: influence of absorbed tumor dose and effect on long-term survival. $J$ Nucl Med. 2017;58:598-604.

19. Lindegren S, Frost S, Back T, Haglund E, Elgqvist J, Jensen H. Direct procedure for the production of ${ }^{211}$ At-labeled antibodies with an epsilon-lysyl-3(trimethylstannyl)benzamide immunoconjugate. J Nucl Med. 2008;49:15371545 .

20. Thurber GM, Wittrup KD. Quantitative spatiotemporal analysis of antibody fragment diffusion and endocytic consumption in tumor spheroids. Cancer Res. 2008;68:3334-3341.

21. Thurber GM, Dane Wittrup K. A mechanistic compartmental model for total antibody uptake in tumors. J Theor Biol. 2012;314:57-68.

22. Nugent LJ, Jain RK. Extravascular diffusion in normal and neoplastic tissues. Cancer Res. 1984;44:238-244. 